\title{
PACIENTES COM HIPERTENSÃO INTRACRANIANA (HIC) E SUAS PECULIARIDADES A SEREM CONSIDERADAS PARA UM TRANSPORTE AEROMÉDICO SEGURO
}

Felipe Trevisan Matos NOVAK ${ }^{1}$, Michelle TAVERNA ${ }^{2}$, Andréa Mendes DE OLIVEIRA ${ }^{3}$, Alessandra Aparecida Tavares NEVES ${ }^{4}$

\section{RESUMO}

A ausência ou demora no reconhecimento e tratamento da hipertensão intracraniana (HIC) pode levar à danos ou sequelas que poderão ser irreversíveis. O manejo assertivo de paciente neurologicamente comprometidos deve evitar complicações secundárias à hipóxia, isquemia e a própria Hipertensão Intracraniana (HIC). Este trabalho se trata de pesquisa bibliográfica, cujo objetivo foi encontrar na produção científica em bases de dados Medical Literature Analysis and Retrieval System Online (MEDLINE) - via Pubmed, sobre os cuidados essenciais no transporte de pacientes com HIC. A equipe deve ter atenção as pequenas alterações hemodinâmicas e para tal precisa de conhecimento, habilidade para correções bem como ter disponíveis materiais, equipamentos compatíveis com 0 voo e medicamentos específicos. Portanto a utilização de aeronaves para o transporte destes pacientes nos parece ser segura desde que sejam observados 0 bom planejamento $e$ atendam as peculiaridades da vulnerabilidade neuro/hemodinâmica que os pacientes de HIC apresentam.

Palavras-chave: transporte aeromédico, hipertensão intracraniana (HIC).

\section{INTRODUÇÃO}

Observando anatomicamente, o cérebro é um órgão de ocupação singular no corpo humano, está posicionado dentro de uma caixa óssea, mergulhado no líquido cefaloraquidiano (LCR) e com sangue circulante. A Pressão Intracraniana (PIC) é definida como a pressão exercida pelo cérebro, pelo sangue e pelo líquor e pode apresentar flutuações, por isso, sua pressão é definida como a pressão de um estado de equilíbrio em relação a atmosfera, sobre o qual se superpõe os componentes cardíaco e respiratório (COLLI, 1990).

De maneira geral, o autor aponta que as principais doenças responsáveis pelo aumento da PIC envolvem o crescimento de lesões que ocupam espaço na caixa craniana, obstrução da circulação liquórica (hidrocefalia), aumento de líquidos no

\footnotetext{
1. Médico de voo Helisul, Membro do Dep. de Neurociência da ABRAERO, Especialista em Transporte e Resgate Aeromédico pela Faculdade Inspirar, Curitiba, Paraná, Brasil. felipenovak@gmail.com

2. Enfermeira de Voo Helisul, $1^{\circ}$ Ten da Força Aérea Brasileira, Presidente ABRAERO, Coordenadora do Dep. de Neurociência da ABRAERO. Curitiba, Paraná, Brasil. michelletaverna@hotmail.com

3. Médica de voo Helisul, Pós-graduanda em Transporte e Resgate Aeromédico da Faculdade Inspirar, Curitiba, Paraná, Brasil. andrea.oliveira80@hotmail.com

4. Enfermeira, Sargento no Centro de Atenção à Saúde (CAIS) da Força Aérea Brasileira Curitiba, Paraná, Brasil. aatneves@gmail.com
} 


\section{CON \\ Cons}

espaço intersticial e/ou intracelular cerebral (edema cerebral) e ingurgitamento venoso (aumento do volume sanguíneo intracraniano) (COLLI, 1990).

Em pacientes neurologicamente comprometidos, o manejo assertivo deve evitar complicações secundárias à hipóxia, isquemia e a própria Hipertensão Intracraniana (HIC). A ausência ou demora no reconhecimento desses fatores levará a danos ou sequelas que poderão ser irreversíveis (GUIMARÃES, et al. 2003).

Para GUERRIERO (2021), os pacientes com distúrbio neurológico e/ou neurocirúrgico, trazem preocupação para as equipes de voo, visto que os riscos de piora clínica e neurológica aumentam exponencialmente caso não se tenha planejamento e transporte adequados, o que poderá resultar na perda ainda maior de funções neurológicas, com sequelas irreparáveis e altos custos sociais.

\section{METODOLOGIA}

Esse trabalho foi uma revisão bibliográfica acerca do transporte aéreo de pacientes com hipertensão intracraniana (HIC). Através da análise crítica e leitura sistemática de livros, sites e artigos científicos selecionados em buscas na base de dados Medical Literature Analysis and Retrieval System Online (MEDLINE) via Pubmed, utilizando as palavras-chave: intracranial hypertension, aerospace medicine, e de bases de dados epidemiológicos como DATASUS. O período do material considerado foi de 1990 a 2021.

\section{RESULTADOS E DISCUSSÕES}

Segundo a doutrina de Monro-Kellie, o volume total do compartimento intracraniano deve ser constante devido a estrutura óssea rígida do crânio. Esse volume é preenchido pelo parênquima cerebral (cerca de $80 \%$ do volume), pelo líquor (cerca de $5-10 \%$ ) e por sangue intravascular (aproximadamente 8-12\%). Se ocorrer aumento de volume de um dos componentes, deverá haver redução dos outros para manter a pressão intracraniana (PIC) constante - em 10-15mmHg - e permitir adequada perfusão do tecido encefálico (HALL, 2011). Portanto, se estivermos frente a uma hemorragia intracraniana volumosa, com acréscimo de mais um conteúdo ao espaço intracraniano, o volume de líquor e de sangue intra-arterial tendem a diminuir para que a PIC se mantenha estável. Porém existe um limite para estes mecanismos e, a partir 


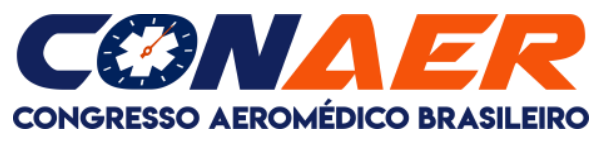

dele, a pressão arterial eleva-se para vencer o aumento da PIC e manter um gradiente entre as pressões arterial e venosa - pressão de perfusão cerebral (PPC) (HALL, 2011).

Para o diagnóstico adequado são necessários exames de alto custo como tomografia computadorizada, ressonância magnética, angiografia cerebral, Doppler transcraniano e eletroencefalograma, no Brasil disponíveis em grandes serviços, o que leva a impreterível urgência na transferência desses pacientes para locais especializados de diagnóstico e tratamento (HERTELENDY et al., 2011).

Baseado nessas prerrogativas compreende-se que o transporte aeromédico de pacientes com HIC a estes centros deva ser feita de maneira rápida e segura de modo a possibilitar que o tratamento seja instituído o mais precocemente possível. Uma das modalidades de transporte mais eficientes para este fim é o transporte aéreo, porém, quando utilizado, o paciente poderá estar sujeito a determinadas variáveis de podem agravar o seu quadro clínico.

Quando se fala em pacientes com comprometimento neurológico, os trabalhos apontam alguns cuidados indispensáveis para maior estabilidade durante o transporte pelo modal aéreo, dentre eles: 1) Cuidado no posicionamento do paciente dentro da aeronave; 2) Elevação da cabeceira do leito em $30^{\circ}, 3$ ) Monitoramento contínuo do paciente; 4) Preferência pelo uso de pista longa; e 5) Redução de manobras bruscas em voo com aceleração e desaceleração suaves da aeronave. $O$ conjunto de tais medidas permite que 0 transporte destes pacientes seja feita de forma segura para centros especializados de tratamento (GUERRIERO 2021).

Para HERTELENDY et al. (2011), os requisitos mínimos para o transporte aéreo incluem, além das descritas: 1) Manejo adequado as vias aéreas, considerando a intubação e manutenção PCO2 entre 25 à $35 \mathrm{mmHg}$ quando em ventilação mecânica. Com reavaliação das funções respiratórias e suplementação de oxigênio conforme necessário; 2) Drogas como manitol e/ou diuréticos osmóticos, corticoides como dexametasona, anticonvulsivantes como diazepam, midazolam, fenitoína e carbamazepina, soluções salinas e glicose devem estar disponíveis para o manejo de intercorrências; 3) Monitorização rigorosa da pressão arterial, com manutenção de níveis inferiores a $150 \mathrm{mmHg}$ de pressão arterial sistólica; 4) Acessos venosos pérvios; 5) Checagem da temperatura corporal; e 6) Monitorização das funções 
neurológicas. Ressalta ainda a importância de ofertar no mínimo as condições presentes na assistência da origem e por isso considerar equipamentos compatíveis com manejo de monitorização de paciente neuro-crítico como ventilador mecânico com diferentes modos ventilatórios, monitor multiparamétrico com módulos que permitam realizar: monitorização cardíaca, monitorização da pressão não invasiva, oximetria de pulso, capnografia, temperatura, pressão intracraniana (PIC); e bombas infusoras em quantidade suficiente que garantam a manutenção de acessos venosos e a infusão adequada de drogas durante todo o transporte (HERTELENDY et al., 2011).

\section{CONSIDERAÇÕES FINAIS}

O aumento da pressão intracraniana necessita de investigação e tratamento em centros com estrutura adequada. Para isso, algumas vezes os pacientes precisam ser transportados por aeronaves.

Nessa modalidade de transporte, os pacientes são submetidos a estressores de voo como forças acelerativas, hipóxia, variações de pressão e temperatura entre outros. Em pacientes com HIC a equipe deve ter atenção as pequenas alterações hemodinâmicas e, para tal, precisa de conhecimento e habilidade para corrigi-las bem como disponibilidade de materiais, equipamentos compatíveis com o voo e medicamentos específicos.

Portanto a utilização de aeronaves para o transporte destes pacientes nos parece ser segura desde que sejam observados o bom planejamento e atenção às peculiaridades da vulnerabilidade neuro/hemodinâmica que os pacientes de HIC apresentam.

A revisão das bases de dados nos forneceu informações a respeito das patologias causadoras da HIC em si, porém o transporte aéreo ainda merece atenção e maiores discussões, visto suas limitadas publicações. 


\section{CONAER \\ CONGRESSO AEROMÉDICO BRASILEIRO}

\section{REFERÊNCIAS}

COLLI BO. Hipertensão intracraniana: Fisiopatologia, diagnóstico e tratamento. jbnc [Internet]. 6o de agosto de 2018 [citado 11ํ de junho de 2021];2(01):30-9. Disponível em: https://jbnc.emnuvens.com.br/jbnc/article/view/1587. acessado em 12 março 2021.

GUERRIERO, MP. Transporte e resgate aeromédico em patologias neurológicas. In: SUEOKA,JS. FREIXO, JAA; e TAVERNA M. Transporte e Resgate Aeromédico. Guanabara Koogan. Rio de Janeiro, 2021.

GUIMARÃES, Jl, et al. Diretriz de doença cardiovascular e viagem aérea: nocões de transporte aeromédico, [Internet]. De 11 de Dezembro de 2003. Disponível em: http://publicacoes.cardiol.br/consenso/2003/site/049.pdf, acessado em 12 março 2021.

HALL JE. Efeitos das forças de aceleração sobre o corpo em fisiologia aeroespacial. Guyton \& Hall - Tratado de fisiologia médica. Ed. 12. Editora Elsevier. 2011.

HERTELENDY et al. Neurologic emergencies. In: Critical Care Transport. Jones and Bartlett Publishers. Massachusetts, 2011. 